\title{
Anderson-Fabry disease: clinical manifestations and impact of disease in a cohort of 98 hemizygous males
}

\author{
K D MacDermot, A Holmes, A H Miners
}

\begin{abstract}
Objectives-To determine the natural history of Anderson-Fabry disease (AFD) as a baseline for efficacy assessment of potentially therapeutic drugs.

Design-The first large cross sectional study of a patient cohort from the AFD clinical and genetic register (UK), maintained for the last 15 years.

Measures-Prevalence, mortality, frequency of AFD manifestations, and impact of disease on patient lives, assessed from the AFD register and the disease specific questionnaire.

Results-The median cumulative survival was 50 years $(n=51)$, which represents an approximately 20 year reduction of life span. Neuropathic pain was present in $77 \%(n=93)$ with mean pain score of 5 (scale 0-10) despite treatment with anticonvulsants and opiates. Pain stopped in only $11 \%$. Cerebrovascular complications developed in $24.2 \%$ and renal failure in $30 \%$. The onset and progression of serious AFD manifestations was highly variable. The relationship of gastrointestinal manifestations on weight, using body mass index $(B M I)$, was significant $(p=0.01)$. High frequency sensorineural deafness was confirmed in $78 \%$ of audiograms. Neuropathic pain and angiokeratoma were absent in five adult males ( $5 \%)$. Median age at diagnosis of AFD was 21.9 years $(n=64)$.
\end{abstract}

Impact of disease-Attendance at school, sports, and social activity were significantly affected by AFD. Only $56.6 \%(n=46)$ of patients were employed. Psychosexual effects of genital angiokeratoma, genital pain, and impotence were not previously recognised.

Conclusion-The majority of males experience multiple disease manifestations and the duration of neuropathic pain was lifelong. The AFD register proved useful for the determination of baseline disease parameters in this cohort. (f Med Genet 2001;38:750-760)

Keywords: Anderson-Fabry disease; natural history; mortality; prevalence

Anderson-Fabry disease is the second most common glycosphingolipid storage disorder (after Gaucher disease) with birth frequency of 1 in $100000 .^{1}$ A deficiency of $\mathrm{X}$ linked lysosomal hydrolase, $\alpha$-galactosidase, leads to the accumulation of uncleaved glycosphingolipids, predominantly globotriosylceramide (Gb3). Data on clinical presentation of AFD consist mainly of reports on individual subjects, large families, or small cohorts investigated by a single speciality. Reporting bias towards the milder and severe ends of the disease spectrum is therefore a possibility.

The clinical hallmark of AFD is neuropathic pain and the histopathological findings of lipid accumulation suggest that the pain is the result of degeneration of nerve fibres in the dorsal root ganglion cells with associated axonal degeneration of the small fibres in pathways subserving pain. $^{23}$ The deposition of glycosphingolipids begins in the lysosomes, causing engorgement of cells, tissue hypertrophy, and eventually cell death and organ failure. Lipid deposits are prominent in the endothelium and the media of small vessels, renal tubules and glomeruli, cardiac muscle and conducting fibres, autonomic ganglia, and in specific cortical and brain stem structures. These histopathological findings explain the protean clinical manifestations of the disease, of which the most significant are renal failure, cardiomyopathy, and multiple cerebrovascular accidents (CVAs) causing premature death. ${ }^{4}$ Mortality data at present quoted for AFD were obtained in 1962 on eight UK families. ${ }^{5}$ Since AFD patients have benefited from renal transplants and effective immunosuppression, these data may now be outdated. Owing to the generalised nature of this inborn metabolic defect, multiple disease manifestations in individual patients were to be expected because $\mathrm{Gb} 3$ accumulation occurs in most non-neuronal tissues and body fluids. Data on the severity and frequency of AFD manifestations in a large cohort would be helpful for clinical management and form the baseline for the assessment of investigational drugs efficacy.

\section{Methods}

ASCERTAINMENT

AFD patients were ascertained in 1985 by contacting all Regional Genetic Units and Enzyme Reference Laboratories in the UK, requesting notification of patients with low $\alpha$ galactosidase level. Clinical details from these patients were obtained through their general practitioners, cardiology, neurology, and renal specialists, hospital records, necropsy records, patient home visits, by patient interviews, patient questionnaire, and our own clinical evaluation. In addition, we have received patients' records from the late Dr D Wise.
24 July 2001

Accepted for publication

31 July 2001 
Table 1 AFD male cohorts examined in the study

\begin{tabular}{|c|c|c|c|}
\hline Source and measures & No of patients & Mean age (years) & $95 \%$ confidence interval (years) \\
\hline Total of patients evaluated in the study (prevalence, disease manifestations) & 98 & 34.8 & $31.69,37.91$ \\
\hline $\begin{array}{l}\text { Subset of patients (attending the clinic) who responded to questionnaire about } \\
\text { psychosocial impact of the disease }\end{array}$ & 46 & 34.5 & $29.08,37.96$ \\
\hline Dead relatives also affected with AFD & 51 & 48.53 & $44.75,55.25$ \\
\hline
\end{tabular}

With the cooperation of the AFD patient support group we were able to evaluate possibly all (or most) patients with a confirmed or suspected diagnosis of AFD in the UK to date. This genetic register has now been maintained for 15 years, and it contains demographic, clinical, biochemical, and DNA data on families from the UK and Eire. These data were obtained from continuous assessment in genetic clinics, which are shared with general practitioners and supplemented by results from individual patients' hospital specialist.

The age at onset and frequency of disease manifestations were obtained from the AFD register (table 1). The cohort identified consisted of 98 patients with confirmed low leucocyte $\alpha$-galactosidase, except in three older patients, in whom the enzyme level was measured in plasma or in fibroblasts. The reference range for leucocyte $\alpha$-galactosidase was $33-134 \mathrm{nmol} / \mathrm{mg}$ protein $/ \mathrm{h}($ mean= $=50)$ and the hemizygote range $0.41-5.0$. However, two males with multiple clinical manifestations of AFD had higher leucocyte $\alpha$-galactosidase values of 5.8 and $12 \mathrm{nmol} / \mathrm{mg}$ protein $/ \mathrm{h}$ respectively.

$\alpha$-galactosidase gene mutation was identified in all families (except one) and consisted of different mutations in each, including nonsense, missense, stop, and exon skipping. ${ }^{6}$ From the cohort of 98 AFD males, 50 were personally evaluated and remain under follow up, apart from eight who have since died. Four patients died before their clinic visit and data on the remaining 44 patients were obtained from their medical practitioners, from telephone interviews, and questionnaires. The mean age of the 98 affected males is shown in table 1 and, of these, 16 were children under the age of 18 .

Patients included in this cohort were those in whom either complete or partial clinical data were available, excluded were those who did not wish to be contacted/be tested/could not be traced. The cohort number of 98 was thus larger than the number of AFD males currently living in the UK (80), as included were patients who had died in the last 15 years and those who live abroad.

MORTALITY DATA

Patients included were those who have a first degree relative alive and who were able to give information on the diagnosis, cause of death, date, and place so that confirmation could be sought from the hospital records/death certificate (table 1). Notifications of the cause of death were received from patients' doctors during the last 15 years.

CLINICAL MANIFESTATIONS OF AFD

Data sought from the AFD register included the onset and duration of neuropathic pain, its severity, and analgesic drug intake for which a McGill pain questionnaire (short form) was used. ${ }^{7}$ This questionnaire consists of questions relating to the location, nature, and intensity of pain, which the patient answers by choosing from a list of options or from grading a scale of $0-10$.

Further, the age of onset of transient ischaemic attacks (TIAs), CVA, renal failure, or dementia were obtained from medical records. Headaches occurring more than once a week were recorded.

We have examined the frequency of self reported hearing loss and tinnitus. Abnormal renal function was defined as serum creatinine $>120 \mu \mathrm{mol} / 1$ or creatinine clearance $<85$ $\mathrm{ml} / \mathrm{min}$ or $\mathrm{GFR}<120 />160 \mathrm{ml} / \mathrm{min} / \mathrm{m}^{2}$. Proteinuria was defined as urine protein $>0.15$ $\mathrm{g} / 24 \mathrm{~h}$ or $>1+$ on Dipstix testing. LVH was defined on ECG as an increased amplitude of the QRS deflection and inverted $T$ waves with ST depression in the left precordial leads, or on echocardiogram as an increased measurement of intraventricular septum in diastole (IVSd) and/or an increased left ventricular posterior wall thickness in diastole (LWd), and/or on cardiac MRI as an increase in LV mass ( $>113$ $\left.\mathrm{g} / \mathrm{m}^{2}\right)$. Cardiac enlargement was assessed in AFD patients by various imaging techniques, which evolved over time, so data on progression of cardiac enlargement were not possible to assess. Heart valve abnormality was taken as that documented on echocardiogram; pulse rate and $\mathrm{PR}$ interval measurements were obtained from two most recent ECG recordings. Lymphoedema was defined as nonfluctuating ankle/calf swelling occurring over a period of hours/days, in which venous thrombosis was excluded by venogram.

Further, patients were asked about chronic abdominal pain (at least once a week), vomiting, and diarrhoea, which was investigated and no pathology was found. Patients were asked to assess whether they consider themselves underweight, experience excessive tiredness (when compared with their peers), or have reduced/loss of sweating. Facial dysmorphology was assessed during clinical examination for the presence of thick lips, bulbous nose, and thickening of nasolabial folds.

The delay in reaching the diagnosis of AFD was estimated in individual patients from the self reported onset of the characteristic AFD rash and from the onset of neuropathic pains. This information was checked with their medical records. The presence/absence/onset of angiokeratoma were recorded in the clinic from 50 patients. Information on the remaining patients relied on findings by patients' physicians. 
AFD SPECIFIC QUESTIONNAIRE

In June 1999, this questionnaire was created, based on pilot in depth interviews with patients and medical practitioners. It was sent to 10 patients as a pilot study to assess the specificity and acceptability of information sought. A modified version of the questionnaire was sent to 59 AFD males attending the clinic of whom 46 agreed to participate and provided data $(77.9 \%)$. It contained questions on marital status, general health, effect of AFD pain and associated manifestations on attendance at school, participation in sports, employment, social activities, psychosexual functioning, and a question rating their medical care. Questions relating to the presence of clinical manifestations were checked against the data on the AFD register. Non-responders were reminded on one occasion. Some participants did not complete all measures and therefore the exact number is reported for each result. Local ethical committee approval was obtained.

STATISTICAL ANALYSIS

A Kaplan-Meier curve, which displays the cumulative probability of a particular end point, was used to examine patients' survival and the current data on UK male general population was provided by the UK Government Actuaries Department using 1997-1999 life tables.

Differences in BMI values between patients with and without gastrointestinal (GI) symptoms were assessed using a Mann-Witney U test as the scores were non-normally distributed.

\section{Results}

The cohorts examined in this study are summarised in table 1. As the mean and median values for age were very similar, only the mean age is shown. The AFD manifestation cohort included all assessable UK patients, the postal questionnaire cohort consisted mainly of patients evaluated in the clinic. Their mean ages were comparable and the questionnaire cohort was perhaps enriched for patients living nearer our clinic, but patients were prepared to travel and families were seen on home visits, so that the demographic differences were not substantial.

\section{MORTALITY}

The survival function in fig 1 shows the median cumulative survival in AFD males to be 50 years, which represents an approximate reduction of 20 years from that of the general population. The mortality curve for AFD males showed a steep decline in survival after the age of 35. The causes of death were usually multiple, predominantly renal failure/CVA. The necropsy report in nine patients included myocardial infarction (MI)/cardiomyopathy and in three the diagnosis of psychosis. One patient died from perforated bowel and one from lymphoma after a renal transplant.

PREVALENCE OF AFD IN THE UK

Prevalence was 1 in 366000 males $(n=80)$, mean age 33.69 years $(95 \%$ CI $30.69,36.69)$.

\begin{tabular}{|c|c|}
\hline $\begin{array}{c}\text { Survival time } \\
\text { (years) }\end{array}$ & Kaplan-Meier curve \\
\hline Mean: 48.53 & $\begin{array}{c}95 \% \text { confidence interval } \\
(45.99-51.07)\end{array}$ \\
\hline Median 50.00 & $\begin{array}{c}\text { 25th-75th centile } \\
(40-56)\end{array}$ \\
\hline
\end{tabular}

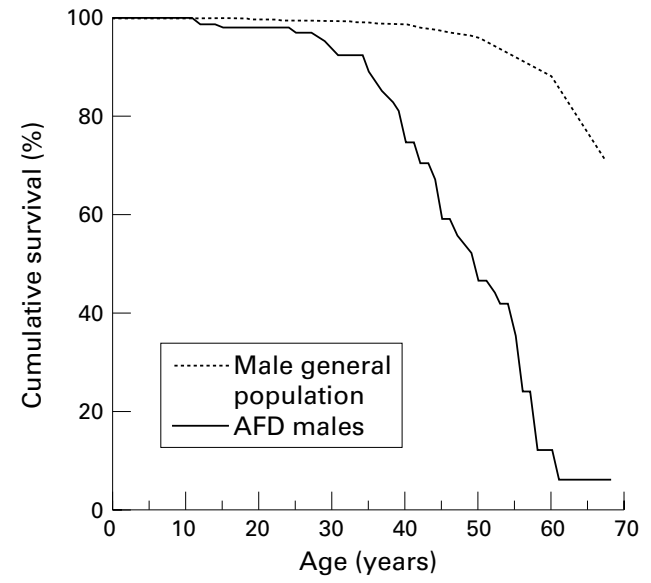

Figure 1 Cumulative probability of death for AFD males using Kaplan-Meier survival tables. The median cumulative survival in AFD males is 50 years, which represents an approximate reduction of 20 years from that of the general population. The mortality curve for AFD males shows a steep decline in survival after the age of 35 .

An additional four males with incomplete documentation were excluded. There was a total of 67 families in which AFD has been documented, containing affected carrier females and affected males. In the majority, AFD was segregating over the last three to four generations, except in 20 males who had no family history of AFD. In half of these, carrier status in their mother was confirmed by clinical examination, leucocyte and hair root $\alpha$-galactosidase estimations, and subsequently by mutation analysis. In the remaining males the origin of the new mutation is at present unknown.

Table 1 shows the groups of patients examined in the study with data on the number of patients, mean age, and confidence interval.

Table 2 shows the mean age of onset of characteristic AFD manifestations (onset of neuropathic pain, angiokeratoma) and of serious complications of the disease. The frequency of disease manifestations in this cohort is shown in table 3. For each parameter, the number denotes the number of patients from whom definite information was obtained and the percentage was calculated from this number. For age at disease manifestation for patients who have since died, this was recorded as age at the patient's last review. Many data were required for many patients and proved difficult to obtain. The total number of patients investigated in tables 2 and 3 therefore vary with each manifestation. 
Table 2 Onset of disease manifestations in AFD males

\begin{tabular}{lll}
\hline Disease manifestation & $\begin{array}{l}\text { Mean age of onset } \\
(95 \% \text { CI) }\end{array}$ & $\begin{array}{l}\text { Total number of } \\
\text { patients investigated }\end{array}$ \\
\hline Neuropathic pain & $10.1(8.6,11.6)$ & 93 \\
Angiokeratoma & $16.8(14,19.6)$ & 73 \\
CVA (excluded are children <18 years) & $40.4(35.5,45.2)$ & 70 \\
TIA (excluded are children <18 years) & $38.8(35.2,42.9)$ & 70 \\
Renal dialysis & $36.7(32.6,40.7)$ & 84 \\
Renal transplant & $40(34.5,45.5)$ & 84 \\
Left ventricular hypertrophy & $42(37.9,45)$ & 34 \\
Self reported hearing loss & $29(26.6,31.4)$ & 61 \\
\hline
\end{tabular}

Data on the onset of characteristic and serious disease manifestations were obtained from patients attending the clinic and from those evaluated by their own physicians. The variable numbers of patients in several groups represent those that were investigated.

Table 3 Frequency of disease manifestations in AFD males

\begin{tabular}{lll}
\hline Disease manifestation & Present in \% patients & $\begin{array}{l}\text { Number of patients } \\
\text { investigated }\end{array}$ \\
\hline Neuropathic pain & 77 & 93 \\
Angiokeratoma & 71 & 73 \\
Hypohidrosis & 56 & 52 \\
TIA or CVA (excluded are children <18 years) & 24 & 70 \\
End stage renal failure (excluded are children & 30.8 & 84 \\
$\quad<18$ years) & 84 & 44 \\
Proteinuria & 47 & 60 \\
Abnormal renal function & 45 & 76 \\
Ankle swelling & 56 & 61 \\
Chest pain/palpitation & 88 & 34 \\
Left ventricular hypertrophy & 29 & 34 \\
Heart valve abnormalities & 14 & 72 \\
Lymphoedema & 69 & 70 \\
GI symptoms & 62 & 61 \\
Fatigue & 56 & 41 \\
Dysmorphic face & 41 & 61 \\
Self reported hearing loss & 78 & 23 \\
Abnormal audiogram & 38 & 60 \\
Tinitus & &
\end{tabular}

The percentage of patients with cerebrovascular involvement is lower than in table 2, as several patients had both TIA and CVA. The numbers of patients represent those who were investigated.

\section{NEUROPATHIC PAIN ( $\mathrm{N}=93)$}

Self reported age of onset was obtained from 81 patients who had ever suffered from pain (12 patients never had pain, see below). The majority developed pain in the median age range of $4-12$, but several at a later age of $17,18,24,25,28$, and 51 . Because of this variable age of onset in this cohort, the frequency of neuropathic pain was calculated from both adults and children; $77 \%$ of patients experienced pain at their present age, range 4-61 years. Triggers for pain onset were: hot weather in $73.1 \%$, physical exercise $53.6 \%$, stress $43.9 \%$, alcohol intake $26.8 \%$, fever $58.5 \%$, and $12.2 \%$ felt that pain had occurred for no obvious reason. Sixty percent are taking anticonvulsants and opiates in the doses they were able to tolerate, the remainder took non-steroidal antiinflammatory drug preparations or no painkillers because of side effects. In addition, $19.5 \%$ used rest, $2.4 \%$ relaxation, and $4.8 \%$ relied on application of cold compresses. There were some universal experiences such as location of pains (in the hands and feet) or the nature of the pain sensations (such as burning), while others experienced idiosyncratic pain patterns. A total of $29.2 \%$ of respondents described their pain as a constant background pain whereas $53.6 \%$ described their pain as both constant background pain coupled with attacks of excruciating pain occurring on average four to six times per year and lasting several days.

Fig 2 shows the percentage of patients with pain stratified for age in addition to pain score "at its worst in the last week" using the McGill pain questionnaire (short form). Fig 2 also shows the numbers of patients from this cohort in each age group. The median pain score in all patients, even those on anticonvulsants, was 5 (on scale 0-10). Over half (65\%) scored between 5 and 9, which is considered as pain severity interfering with daily living.

In $11 \%$ of patients the pain has stopped, at a mean age of 24 years, age range 12-35. A total of $12.9 \%$ (12 patients) had never had neuropathic pain. Four were under 15 years and the pain may still develop, three patients were aged 17,24 , and 26 , and the age range of the remaining five patients was 44-58.

Children usually complained of sensitive, painful feet and hands during common childhood pyrexial illnesses although these responded to simple analgesics. With time, the pain intensity increased, and attacks occurred more frequently, during sports sessions and also without overheating or pyrexia. At this time, children were often hospitalised and

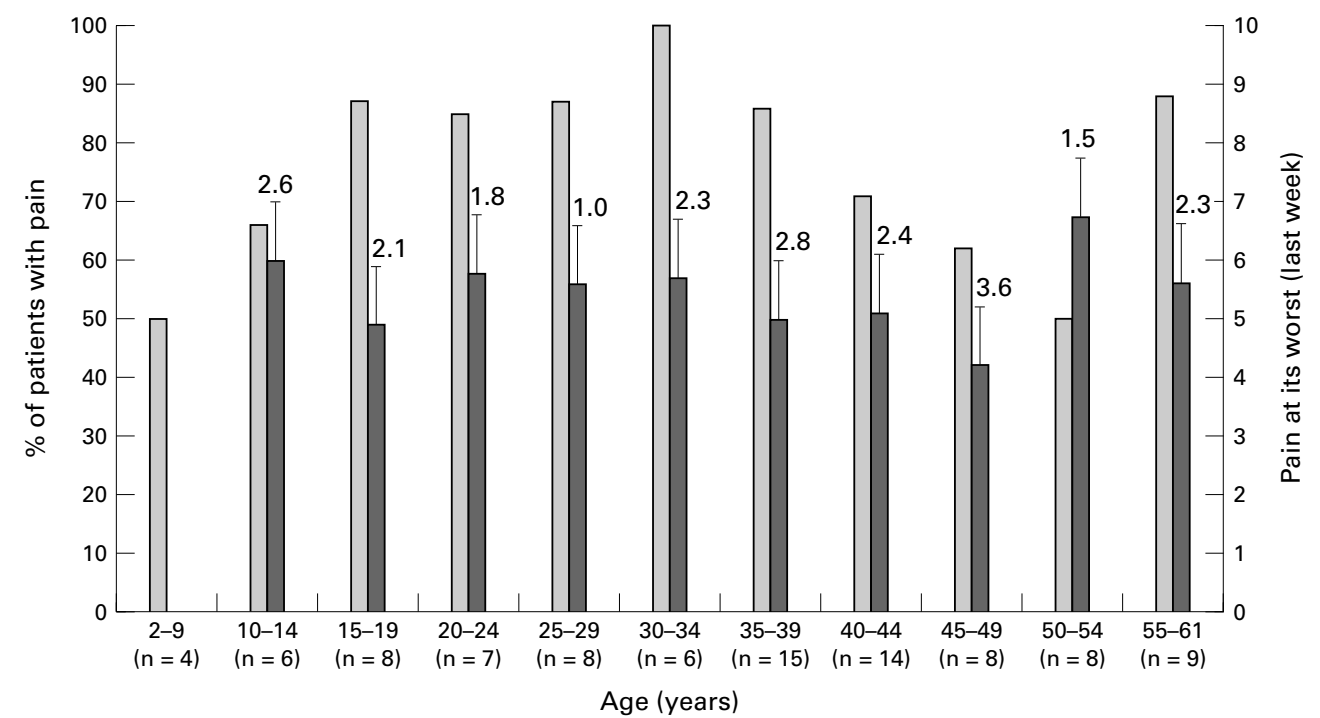

Figure 2 The light grey bars show the percentage of patients with pain and the dark grey bars show their pain scores with standard error of the mean obtained from McGill pain questionnaire. $n=$ total patients investigated in each age group. 
required morphine for acute attacks and anticonvulsants for chronic pain relief.

TIAs and CVA have not so far been reported in children and in this study those under 18 were excluded. Table 2 shows the age of onset separately for TIA or CVA in adults. Table 3 shows the frequency of either, as TIA often preceded CVA and both were often present in the same patient. One male had CVA at the age of 18 and one developed unilateral blindness owing to central retinal artery thrombosis followed by multiple strokes. Treatment with antiplatelet agents was initiated in most patients, but did not appear to prevent further CVA; however, formal analysis was not done. Dementia was present in $18 \%$ and was associated in all cases with recurrent CVAs or TIAs. Headaches were reported by $15 \%$ of patients and were also associated with TIAs and CVAs, except in one case with typical migraine attacks. Two males $(n=97)$ had mild learning difficulty of unknown cause.

RENAL DIALYSIS AND RENAL TRANSPLANT

End stage renal failure (ESRF) in childhood is very rare (it has occurred in one 15 year old, personal communication) and for the frequency calculation in this study, children under 18 were excluded (table 3 ). The ages at onset of renal dialysis and for renal transplant are presented separately in table 2 . One male in this cohort presented with ESRF at the age of 18.

RENAL FUNCTION (TABLE 3)

The age of onset of ESRF showed considerable inter- and intrafamilial variation. The following case summaries illustrate the spectrum of AFD manifestations. Three patients, who died recently aged 44,55 , and 61 , had normal renal function tests shortly before death. The 44 year old patient with a frameshift mutation ( $1 \mathrm{bp}$ deletion at nucleotide 520) and a leucocyte $\alpha$-galactosidase level of $3.6 \mathrm{nmol} / \mathrm{mg}$ protein $/ \mathrm{h}$ had severe cardiomyopathy, coronary artery disease, multiple CVA, and neuropathic pain, but no angiokeratoma. His cause of death was coronary insufficiency. The residual enzyme level is $7.2 \%$, which is in the mild "cardiac variant" range. ${ }^{8}$

There was also interfamilial variation. His identical twin brother died suddenly at 34 with the same manifestations, but a 30 year old cousin has neuropathic pain treated with anticonvulsants, angiokeratoma, which was first confirmed in the clinic at the age of 28 , severe abdominal pains, mild cardiomyopathy, and deteriorating renal function. These members of the same family were discordant for renal function and angiokeratoma. The 55 year old patient with a new missense mutation (R112C) (enzyme result is not available) was diagnosed because of angiokeratoma at the age of 33 and was followed up for 20 years by a renal unit, but his renal function remained normal. He had no neuropathic pains or any other symptoms and worked as a builder until the age of 52 when he presented with recurrent strokes and dementia. The 61 year old patient had an IVS $5+2 \mathrm{~T}>\mathrm{C}$ mutation in exon 6 and a leucocyte $\alpha$-galactosidase level of $1.2 \mathrm{nmol} / \mathrm{mg}$ protein $/ \mathrm{h}$. The residual enzyme level is $2.4 \%$, which is in the mild "cardiac variant" range. ${ }^{8} \mathrm{He}$ had extremely severe neuropathic pains from very early childhood, widespread angiokeratoma, and mild cardiomyopathy. He suffered from extremely severe abdominal pains and died of a perforated bowel, but his renal function remained normal. His brother died from renal failure aged 50 . His nephew has severe neuropathic pains, cardiomyopathy, ESRF, and CVA at the age of 32. Patients in this family showed discordance for renal involvement. These case histories illustrate the variable progression of AFD pathology in individual patients, and show that one or more characteristic symptoms/signs may be absent in individual patients without this being "atypical". ${ }^{9}$ One 5 year old boy from this cohort presented with idiopathic nephrotic syndrome of childhood. His renal biopsy showed changes of focal segmental glomerulosclerosis and histopathological features of AFD were absent.

\section{CARDIAC FUNCTION}

Very few patients under the age of 20 had investigations performed, so the age of onset of cardiomyopathy in this cohort could not be determined. There was a tendency towards older patients having increasingly severe concentric left and right ventricular hypertrophy and enlarged left atrium, but there were also males at the age of 35-40 with normal echocardiograms. The increase in IVSd and LWd values above the normal range were usually the first abnormalities to be detected.

Heart valve abnormalities detected on echocardiogram consisted of mild to moderate mitral regurgitation and thickening of the aortic valve. One patient had mild coarctation of the aorta and congenital bicuspid aortic valve and another patient had ASD.

PR interval mean value was $139.38 \mathrm{msec}$ (95\% CI 131.93, 146.83) $(n=32)$, which is within the normal range of $120-210 \mathrm{msec}$. An additional patient had atrial fibrillation and two had supraventricular ectopics. Common ECG abnormalities were those of $\mathrm{LVH}$ and two patients had partial left and right bundle branch block respectively. One patient had successful ablation for Wolf-Parkinson-White syndrome.

The mean pulse rate was $59.25 \mathrm{bpm}(95 \%$ CI $56.53,61.97)$, median 57 , but a relatively slow pulse rate was observed in older males and in those with significant cardiomyopathy. The lowest recorded pulse rate was $41 \mathrm{bpm}$ in a 37 year old male with severe cardiomyopathy and aortic and mitral valve regurgitation. Coronary artery involvement was documented in only three patients $(n=32)$. One had an inferior perfusion defect on thallium scan, suggestive of ischaemia, one had partial single vessel stenosis, and one had coronary triple bypass. One patient died suddenly postoperatively (renal transplant) from coronary thrombosis. Renovascular hypertension was present in only one patient. 


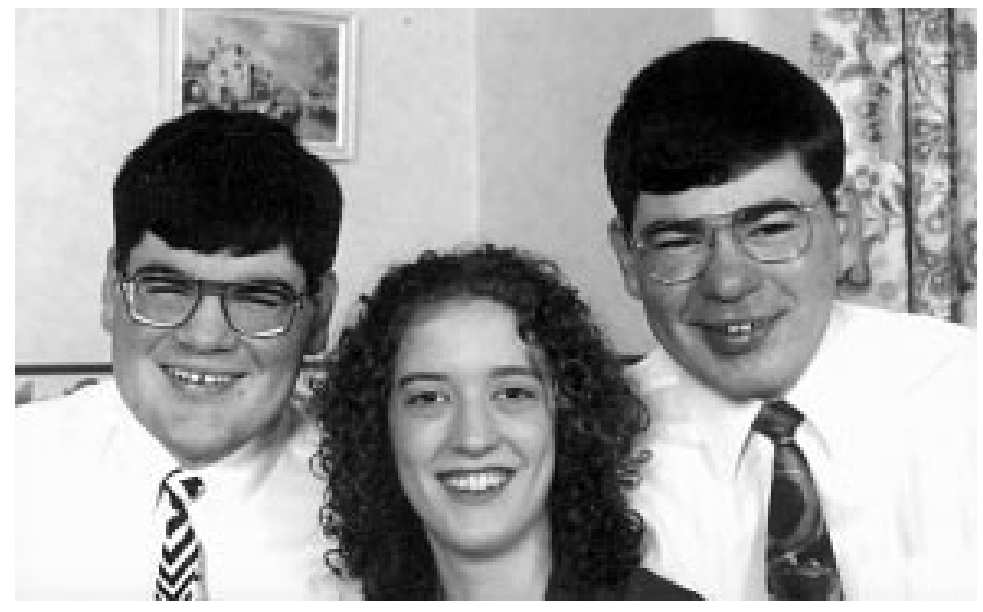

Figure 3 Facial features in two brothers with AFD and their unaffected sister, showing thickening of the lips and nasolabial folds in the affected males.

GASTROINTESTINAL FUNCTION

GI symptoms consisted of recurrent bouts of abdominal pain, described as colic with burning pain, located in the mid and lower abdomen. Nausea, vomiting, and feeling of abdominal distension were common and some patients experienced superficial abdominal skin tenderness. GI symptoms tended to occur after meals and often patients were afraid to eat as a result of these symptoms. All symptomatic patients had numerous investigations, including barium meal, gastroscopy, and colonoscopy, which were all normal. One symptomatic patient had partial colectomy for volvulus and one had colostomy, eventually dying of a perforated large bowel, which showed extensive diverticular disease. GI symptoms occurred as early as 12 years, and were commonly cited as a main symptom of AFD (with pain) in males aged between 30 and 50 years. One 49 year old man was house bound because of up to 20 episodes of watery diarrhoea per day.

Forty-seven percent $(n=57)$ of patients considered themselves underweight. Body mass index (BMI) is a validated measurement for the evaluation of obesity, with a normal range of 19-25. ${ }^{10}$ We used BMI to assess weight in AFD males. BMI was calculated from the baseline weight of 20 patients who have joined the enzyme replacement trial. Twelve had significant GI symptoms and their mean BMI was 18.68 (95\% CI 16.96, 20.40). Eight patients were asymptomatic and their mean BMI was 25.40 (95\% CI 22.54, 28.26). A MannWhitney test analysis of these two groups' results showed this difference to be statistically significant $(p=0.01)$. This result suggests that GI symptoms are associated with a lower BMI score.

OTHER MANIFESTATIONS OF AFD

A degree of hearing loss was noted during clinical review in several patients and was subsequently included in the panel of direct questions.

Audiograms showed mild to severe high frequency sensorineural hearing loss, bilateral or unilateral at 2-3 $\mathrm{kHz}$ range. Three patients without a family history of deafness had confirmed severe sensorineural and conductive hearing loss diagnosed below the age of 10 years and all wore hearing aids. One patient had unilateral cholesteatoma diagnosed in childhood. Tinnitus consisted of brief episodes starting between the ages of 12 and 15 years and continuing to adulthood with varying degrees of severity. Delayed puberty and short stature in childhood were documented in three patients $(n=70)$. Only two patients $(n=93)$ have presented with hand arthropathy in childhood.

AFD diagnosis was made following the onset of neuropathic pain and angiokeratoma. Asymptomatic enlargement of the interphalangeal joints was a frequent finding in older males, but was not formally assessed.

Excessive fatigue was reported by adults but was not present in children.

Dysmorphic facial features were present on careful inspection in about half of the patients evaluated (fig 3) and, when present, were recognisable at 12-14 years of age. Even following years of regular clinic assessments of AFD, the facial features in some patients were uncertain and, in close to half of them, dysmorphic facial features were judged to be absent. Subjective hypohidrosis was reported by $56 \%(n=52)$ of adults and was not present in children. Partial and apparently total body hypohidrosis was observed and tended to occur in older age groups, resulting in overheating and, in some patients, in extreme temperature intolerance during the summer months.

The presence of angiokeratoma corporis diffusum, the characteristic rash of AFD, was assessed in 50 patients in the clinic and in the remaining patients by the patients' hospital specialists. Table 2 shows the age of onset and table 3 the frequency in this cohort. In the majority of patients, the median age of onset was 14-16 years, but early onset (umbilical rash at birth) and late onset of sparse lesions at the age of 28 was also present. The lesions consist of increasing numbers of superficial, dilated capillaries which are non-blanching on pressure, become raised, may bleed, become dark red, keratinised, and fall off. These lesions develop slowly in the "bathing trunk" area, particularly on the dorsum of the penis, scrotum, buttocks, inner thighs, and the back. With age, the lesions become numerous and spread, to be clearly visible on the lips, finger tips, palms of the hands, and toes. Interestingly, five adult males aged 25 to 56 were observed in this study with complete absence of these characteristic lesions, but with other manifestations of AFD.

Age at diagnosis was obtained from 64 patients (mean 21.9, 95\% CI 19.09, 24.71). In 19 patients, the age at diagnosis was unknown and in 15 patients the diagnosis was elective in the form of a prenatal test. As the neuropathic pain attacks and angiokeratoma are characteristic of $\mathrm{AFD}$, the time lag to diagnosis was obtained.

Delay in diagnosis of AFD from the onset of neuropathic pain $(n=39)$ was a mean of 8.18 years $(95 \%$ CI $5.57,10.79)$ and from the onset of angiokeratoma $(n=53)$ was a mean of 10.70 years $(95 \%$ CI $7.97,13.43)$. The diagnosis of 
AFD was often made incidentally and the recognition of cornea verticillata and angiokeratoma by the ophthalmologists and dermatologists, respectively, was high.

PSYCHOSOCIAL PARAMETERS

Data were obtained from the AFD specific questionnaire.

\section{General health and marital status}

None of the patients had cancer, diabetes, or other additionally diagnosed disorders. Three patients $(6.8 \%$ from $n=46)$ had had myocardial infarction in the past. Only $42 \%$ of the group $(n=46)$ were married and two of these were separated or divorced.

\section{Education}

Thirty percent $(n=34)$ of patients failed to obtain the basic school education certificate $(\mathrm{O}$ levels or GCSEs) but $35 \%$ obtained the advanced school certificate (A levels). The results of AFD boys compared favourably with the UK male population in the year 1979/80 $(n=460000)$, as $49 \%$ failed to obtain O levels and $17 \%$ obtained A levels (Annual Abstract of Statistics, Central Statistical Office, 1982 edition, No 118, pp 70 and 129).

A total of $80.9 \%$ of patients stated that AFD affected their attendance at school, and a similar number of patients $(83.3 \%)$ reported that AFD affected their ability to participate in sports.

\section{Employment $(n=46)$}

Eight patients $(17 \%)$ stated that they had never had a job because of the diagnosis of AFD. Only $56.8 \%$ were currently employed. A total of $70.2 \%$ of patients stated that AFD pain interfered with their job and necessitated taking time off work. Other manifestations of AFD, for example, diarrhoea and fatigue, were reported by $68.5 \%$ patients as interfering with their ability to carry out a job.

Social activities $(n=46)$

A total of $36.3 \%$ reported that their social activities were specifically limited by pain and a further $26.4 \%$ felt that social activities were limited by the range of other AFD related symptoms; $9.1 \%$ of patients stated that they had not gone out to enjoy themselves at all.

Psychosexual $(n=46)$

A total of $73.8 \%$ reported a rash on their genitals and $64.4 \%$ were embarrassed by it. A small group (16\%) reported genital pain. These have affected enjoyment of sex for over half the sample $(55 \%)$ and reportedly affected both libido (48.7\%) and self esteem (68.3\%). Nearly two-thirds $(61.5 \%)$ of patients noted that these manifestations of AFD resulted in a fear of initiating sexual relationships, particularly in the teenage years.

Medical care ratings $(n=46)$

Care ratings were alarmingly poor. Just under half rated the information provided at the time of diagnosis as poor (46\%), $45 \%$ noted that genetic counselling had been poor, and a quarter of the sample (24\%) felt that subsequent medical care was poor. Nearly one in two respondents felt that their doctor's knowledge of AFD at follow up was still inadequate (46\%).

The questionnaire concluded with an enquiry as to whether in principle the respondents would be prepared to participate in enzyme replacement trials. The response was on the whole positive and $80.6 \%$ reported feeling optimistic about the future. This was most likely related to this survey and to the potential introduction of therapy.

\section{Discussion}

Recent reports addressed the questions of genotype-phenotype correlation in AFD, the classification of the phenotype into classical, cardiac variant, or atypical, and age stratification into childhood onset, quiescent, and active phase of serious manifestations. ${ }^{11-13}$ Residual enzyme levels are associated with milder phenotypes in a number of metabolic diseases and AFD patients with residual $\alpha$-galactosidase activity $(\sim 1 \%-\sim 10 \%)$ were described as essentially asymptomatic or having a milder form of the disease limited to cardiac involvement. ${ }^{8}$ Biochemical studies in atypical hemizygotes with residual enzyme activity showed the mutant enzyme to have increased or decreased stability and to have higher levels in various tissues than in plasma. Residual plasma or leucocyte $\alpha$-galactosidase activity is therefore not a reliable predictor of phenotype and, in addition, the mean reference enzyme values differ between laboratories. Detailed genotypephenotype correlation studies failed to predict the phenotype on the basis of type or location of the mutation ${ }^{12}$ and was not addressed in this study. Age stratification for frequency of disease manifestation requires a large cohort of patients and comprehensive evaluation, which is difficult to obtain. The aim of this study was to determine the natural history of the disease, which may contribute towards phenotype classification and assessment of therapy.

This is the first study of a large AFD cohort of unselected hemizygous males. The results show progressive and multiple AFD manifestations in patients and significant psychosocial impact of the disease on patients as a group. There appears to be only one other comprehensive cohort study performed in 1962. The authors ${ }^{5}$ studied eight UK families, reporting very accurate clinical histories documented in nine males and two females, and since then many case reports have been published. The frequency of some serious manifestations were studied in relatively small cohorts and these findings were comprehensively reviewed by Desnick et al. ${ }^{412}$

The only mortality data from unselected patients were from $1962^{5}$; the mean age at death was 42 years $(n=19$, range $30-54)$. The mean age at death in AFD males without renal failure, reported in 1967, was 41 years $(\mathrm{n}=94){ }^{14}$

In this cohort, the median cumulative survival time in 51 unselected patients was 50 
years, documenting substantial reduction in life span in AFD when compared to the general male population. Premature death is therefore still evident in AFD despite renal dialysis and transplantation.

The UK prevalence of 1 in 366000 males appears to be an underestimate, as the birth frequency of AFD is 1 in $100000 .{ }^{1}$ This finding can be explained by the current practice of not testing asymptomatic boys at risk for AFD unless medically indicated. Further, some young males did not wish to be tested and the remainder may have mild expression of the disease, so they remain undiagnosed. Approximately two-thirds of patients had a family history of the disease and one-third were new mutations, which agrees with other $\mathrm{X}$ linked disorders.

Pain is recognised as the most severe and debilitating symptom of AFD. The onset is in childhood and in 10-20\% the pain stops with advancing age. ${ }^{4}$ Substantial pain relief is obtained with diphenylhydantoin ${ }^{15}$ and carbamazepine, ${ }^{16}$ but painful crises often require parenteral or oral morphine. ${ }^{17}$ In the present report, pain commonly started in childhood, but onset of pain in adults was documented in several patients. These data differ from current assumptions that an AFD child without pain will not develop it later in life and that the frequency of pain should be estimated only for adults. Pain stopped only in $11 \%$ of patients at a relatively early age. The majority of patients reported neuropathic pain of life long duration with high levels of residual pain despite treatment with as high doses of anticonvulsants as they could tolerate (fig 2). This finding suggests that pain relief remains inadequate with this class of drugs. In five adults with other systemic manifestations of AFD, the pain and angiokeratoma were absent and biochemical study of the mutant enzymes would be of interest. Increased perception thresholds to warm and cold temperatures in males and carrier females were shown to initiate burning pain and acute discomfort. ${ }^{18}$ Objective evaluation of pain and paraesthesias can be performed by constant current perception testing ${ }^{19}$ and by skin biopsy findings of severely reduced small myelinated and unmyelinated nerve fibres. ${ }^{20}$ However, these investigations are nonspecific and would not be diagnostic of AFD in patients with unexplained pain. Eight patients in this cohort never had neuropathic pain although in all some other manifestations of AFD are present. The aetiology of this apparent sparing of neurological tissue involvement is not understood.

In one study, the mean age at onset of cerebrovascular complications was 33.8 years $(n=43)$ and the commonest anatomical localisation for CVA was at the vertebrobasilar territory with high recurrence rate and with death. ${ }^{21}$ Another cohort study ${ }^{22}$ of 33 males reported the incidence of cerebrovascular complications as $24 \%$, with lesions localised to both the carotid and vertebrobasilar territories, and occurring mostly before the age of 40 . Our data broadly agree with previous reports. The youngest patient presented with CVA at the age of 18 before the diagnosis of AFD was made. A similar case was reported as "atypical", ${ }^{23}$ because of young age at presentation, but subsequently both these patients have developed the characteristic multiple manifestations of AFD. The frequency of TIAs in this report is almost certainly an underestimate, as on subsequent direct questioning, several patients have admitted to attacks of diplopia, ataxia, or hemisensory loss, not reported to their medical practitioners. This observation is supported by the findings of diffuse neuronal involvement, shown by proton MRS measurements of $\mathrm{N}$-acetylaspartate, which extended beyond the areas of MRI visible cerebrovascular abnormalities even in asymptomatic males. ${ }^{24} 25$

Dementia in all our cases was associated with recurrent TIAs or CVAs. Psychiatric disorders in this chronic and painful disease are not surprising; $20 \%$ of males in this cohort scored within the range of probable clinical diagnosis of anxiety and depression, with a further $20 \%$ having suggestive symptoms, and two have contemplated suicide (unpublished data). Similar findings have previously been reported by Grewal. ${ }^{26}$ Attacks of dizziness and vertigo were frequently reported in this cohort and were mostly diagnosed as being of vestibular origin on caloric testing.

The proportion of AFD males who develop ESRF is unknown. In $83 \mathrm{AFD}$ patients reported to the European renal dialysis and renal replacement registry, the mean age at dialysis/transplant was 38 years. ${ }^{27}$ Their survival on dialysis was shorter than for other primary nephropathies, mainly because of cardiovascular complications, but renal graft survival was comparable. The onset of proteinuria is probably between the ages of 10 and 20 years, but early changes on renal biopsy were observed in males in this age group even in the absence of proteinuria or hypertension. ${ }^{28}$ These early lesions consisted of diffuse glycolipid accumulation in every glomerular, vascular, and interstitial cell and irregular deposition in the distal convoluted tubules and loops of Henle. The accumulation of predominantly Gb3 in the kidneys was age dependent; studies of necropsy specimens have shown a 6-10 fold increase in 30-40 year old males. ${ }^{4}$ Urine sediment showed an increased number of renal tubular cells containing an up to 30 fold rise in Gb3. ${ }^{29}$ Published case histories commonly report gradual onset of renal failure over several or many years, but the disease may present with ESRF. ${ }^{30}$ In this cohort, one patient presented with ESRF at 18 years and several patients aged 35-55 showed previously undiagnosed proteinuria at an insurance medical examination. Several of these had rapidly progressive renal failure subsequently.

Only half of the patients in this report had renal assessment, mainly because the generalised nature of AFD was not appreciated. Renal transplantation in this cohort was associated with perioperative cardiac deaths and the development of lymphoma because of long term immunosuppression. The case histories of three patients with normal renal function at 44,55 , and 61 illustrate variable progression of 
renal involvement between unrelated patients and also lack of genotype-phenotype correlation in their relatives. Two of these patients had residual enzyme levels in the $1-10 \%$ range, which did not correlate with the previously reported mild "cardiac variant" phenotype. This matter requires further study.

Data are available from several cohort studies, documenting a high frequency of LVH, concentric apical hypertrophy, intraventricular septum thickening, widening of the aortic root, mitral valve prolapse, and atrioventricular (AV) conduction abnormalities. ${ }^{4}$

The frequency of $\mathrm{LVH}$ and mitral valve prolapse determined by ECG or echocardiogram was about $60 \%$ and they were already present in one study at a mean age of 15 years. ${ }^{31}$ Goldman et $a l^{\beta 2}$ reported age related progression of echocardiographic LVH changes in young males $(n=23)$ aged $14-43$, which also correlated with their disease severity. Complete AV block and permanent insertion of a pacemaker were also reported. ${ }^{33}$

From the 39 patients evaluated in this study, $88 \%$ had cardiomyopathy but data are missing from the rest of the cohort as asymptomatic patients were not investigated. It is of interest that several males were also documented in the 35-40 age group with normal echocardiogram and ECG parameters and these should be investigated further. The pulse rate appeared to correlate with age and with the severity of cardiomyopathy, but was not formally analysed. Coronary artery disease and myocardial infarction (MI) was confirmed only in three patients. As these are medium sized vessels, their primary involvement in AFD may be overreported. ECG changes of widened QRS complexes, deep Q waves, and abnormal ST-T segment in leads $V_{4}$ and $V_{5}$ may lead to misinterpretation of $\mathrm{MI} .^{32}{ }^{34}$ As chest pain and palpitations are common in AFD patients (56\% in this cohort), the analysis of cardiac enzymes and coronary angiography is indicated, before the diagnosis of MI is made. Sudden or perioperative death could also be the result of arrhythmia or coronary vasospasm. ${ }^{35}$ A convincing case of the "cardiac variant" of AFD was described by Elleder et $a l^{\beta 6}$ in a previously undiagnosed patient who died at the age of 63 with cardiomyopathy owing to Gb3 deposition. Importantly, Gb3 deposition in non-cardiac tissues was absent. The two unrelated hemizygotes described by Nagao et $a l^{37}$ presented after 50 years of age with cardiomyopathy and had high residual enzyme activity in fibroblasts. The absence of non-cardiac AFD manifestations at the age of 50 is unusual and these cases can be described as "cardiac variants " on the basis of absent clinical signs. Monosymptomatic patients under the age of 50 and those with residual enzyme levels of $1-10 \%$ of normal cannot be classified as "cardiac variants" as patients $<50$ years may still develop AFD complications and residual enzyme level is not a reliable predictor of disease severity (see Renal function cases).

The age at onset and the frequency of GI manifestations was reported in a cohort of 41 AFD patients. ${ }^{38}$ Vague abdominal discomfort and cramps started in AFD as early as $5-20$ years in males and 15-35 years in females, and $69 \%$ of males and $29 \%$ of female carriers had progressively incapacitating GI symptoms, including profuse watery diarrhoea, vomiting, severe postprandial colicky abdominal pain, weight loss, and cachexia. In this report, a comparable $69 \%$ of males had GI symptoms, often cited as the main symptom of AFD. GI complications included widespread diverticulitis, volvulus, and a fatal bowel perforation. BMI compared in symptomatic and asymptomatic groups of patients showed significant differences $(p=0.01)$. Previous histological studies showed normal intestinal mucosa, with accumulation of glycolipid in the submucosal autonomic ganglia, neurones of myenteric plexuses, and blood vessels. Findings from radiological studies are age dependent and consist of neuropathic and ischaemic changes, which include thickening of the mucosal folds, loss of large bowel haustration, diverticula, and delayed gastric emptying. Malabsorption is absent, presumably because of the intact jejunal villi. ${ }^{4}$ We conclude that GI symptoms in AFD patients are significant and often remain unrecognised, which precludes the administration of symptomatic treatment and early recognition of complications.

The other most frequently reported manifestations in this study were angiokeratoma (71\%), fatigue (62\%), hypohidrosis (56\%), sensorineural hearing loss $(78 \%)$, and tinnitus $(38.3 \%)$. The frequencies of these manifestations have not been studied previously in a large group of patients. As individual physicians usually see small numbers of AFD patients, it is often assumed that hypohidrosis is invariably present in adults. As hypohidrosis is a subjective symptom and only $56 \%$ of patients at a mean age of 34 reported abnormal/lack of sweating, this result is reported here. This finding can be confirmed or refuted by further study of another cohort. The absence of angiokeratoma and neuropathic pain, found in $5 \%$ of patients, made the diagnosis of AFD difficult, particularly in sporadic cases. Fatigue is a significant symptom of AFD and has been reported in other metabolic disorders; its aetiology is uncertain. In several case reports, sensorineural deafness was described $^{39}$; the results from this cohort show that hearing loss is frequent and high tone deafness, which was usually not clinically significant, was confirmed by audiograms and appeared to be associated with age. AFD facial features were present in this cohort in over half of AFD males and superficially resembled those of acromegaly, as reported by Tsukada et al. ${ }^{40}$ Following an encounter with a few manifesting patients, this "AFD face" was easily recognisable by clinic staff and patients themselves.

Arthropathy of the hand joints was noted in a number of older patients, but was not formally assessed. Similarly, pulmonary function was not investigated. Three patients from this cohort were treated with bronchodilators and one had extensive emphysema with recurrent pneumothorax. 
The diagnosis of AFD in this cohort was made relatively late, at a median age of 19.5 years, on average eight and 10 years after the development of neuropathic pain and angiokeratoma, respectively. This delay has a serious impact on patients' lives and often creates antagonism towards the medical profession. The main reasons for late diagnosis are relatively low awareness of this disease among general physicians and the unusual pattern of episodic pain with heat intolerance or a painless rash on the genitalia, which the patient may wish to conceal and the doctor does not always examine. In cases of familial AFD, the diagnosis was often made in the previous generation, but genetic counselling was not provided for the offspring.

The psychosocial impact of AFD was not studied previously. Over half of the adult males from this cohort never married and continued to live with their parents in a protective environment. The disease did not appear to interfere with their educational achievements but had a major impact on their jobs and promotion prospects. Data on employment in AFD have not previously been reported. The psychosexual effects of angiokeratoma and genital pain have not previously been recognised.

A proportion of AFD males became disabled and dependent at an early age, when residential care is either not available or unsuitable. Males with AFD recorded significantly lower Health Related-Quality of Life scores compared with general population scores $(p=0.0001){ }^{41}$ The Mental Component of Short Form-36 health survey score was significantly lower in AFD males than in males with severe haemophilia $(\mathrm{p}<0.05) .^{42}$ These previously unreported results may be useful for assessing the cost effectiveness of investigational drugs for patients with AFD.

As in other rare metabolic diseases, the disease register and detailed assessment becomes important for learning about the disease and for formulating treatment strategies. Results from enzyme replacement trials are encouraging and include the reduction in accumulated $\mathrm{Gb} 3$ in renal and cardiac tissue, stabilisation of renal function, and the reduction of pain. ${ }^{8}{ }^{43}$ Another potentially therapeutic approach is substrate deprivation by inhibitors of glycosphyngolipid synthesis. ${ }^{44} 45$

In summary, this report documents for the first time the onset and frequency of AFD disease manifestations in a large cohort. The disease manifestations were often multiple, with individually variable progression. New findings were sensorineural deafness, psychosocial impact of the disease, UK prevalence, and current mortality data.

We are grateful to the AFD support group and to the families for their cooperation and to Transkaryotic Therapies Inc, Boston, USA for financial support of AH. Professor Winchester at the Enzyme Laboratory and Molecular Genetics Laboratory, Institute of Child Health, London analysed most of the samples.

1 Meikle PJ, Hopwood JJ, Clague AE, Carey WF. Prevalence of lysosomal storage disorders. $\mathcal{F A M A}$ 1999;281:249-54.
2 Kahn P. Anderson-Fabry disease: a histopathological study of three cases with observations on the mechanism of production of pain. $\mathcal{F}$ Neurol Neurosurg Psychiatry 1973;36: 1053-62.

3 Gemignani F, Marbini A, Bragaglia MM, Govoni E. Pathological study of the sural nerve in Fabry's disease. Eur Neurol 1984;23:173-81.

4 Desnick RJ, Ionnou Y, Eng CM. Fabry disease: alpha galactosidase A deficiency. In: Scriver CH, Beaudet AL, Sly WS, Valle $\mathrm{D}$, eds. The metabolic and molecular bases of inherited disease. New York: McGraw Hill, 1995:2741-84.

5 Wise D, Wallace HJ, Jellinek EH. Angiokeratoma corporis diffusum. $Q \mathcal{F}$ Med 1962;XXXI:177-212.

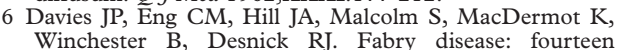
Winchester B, Desnick RJ. Fabry disease: fourteen
alpha-galactosidase A mutations in unrelated families from the United Kingdom and other European Countries. Eur $\mathcal{F}$ Hum Genet 1996;4:219-24.

7 Melzack R. The short-form McGill Pain Questionnaire. Pain 1987;30:191-7.

8 Eng CM, Banikazemi M, Gordon RE, Goldman M, Phelps R, Kim L, Gass A, Winston J, Dikman S, Fallon JT, Brodie S, Stacy CB, Mehta D, Parsons R, Norton K, O'Callaghan M, Desnick RJ. A phase $1 / 2$ clinical trial of enzyme replacement in Fabry disease: pharmacokinetic, substrate clearance, and safety studies. Am f Hum Genet 2001;68: 711-22.

9 Elhadad S, Melet V, Domniez T, Cosson S, Chollet D, Vedel J. Complete atrioventricular block disclosing Fabry's disease (in French)]. Arch Mal Coeur Vaisseaux 1997;90: 393-7.

10 Dickey RA, Bartuska D, Bray GW, Callaway CW, Davidson ET, Feld S. AAC/ACE position statement on the prevention, diagnosis, and treatment of obesity (1998 revision). Endocr Pract 1998;4:297-310.

11 Ashton-Prolla P, Tong B, Shabbeer J, Astrin KH, Eng CM, Desnick RJ. Fabry disease: twenty-two novel mutations in the alpha-galactosidase A gene and genotype/phenotype correlations in severely and mildly affected hemizygotes and heterozygotes. F Invest Med 2000;48:227-35.

12 Desnick RJ, Ioannou YA, Eng CM. Alpha-galactosidase A deficiency: Fabry disease. In: Scriver C, Beaudet A, Sly W, Valle D, Kinzler KE, Vogelstein B, eds. The metabolic and molecular bases of inherited diseases. New York: McGrawHill, 2001:3733-74

13 Eng CM, Resnick-Silverman LA, Niehaus DJ, Astrin KH, Desnick RJ. Nature and frequency of mutations in the alpha-galactosidase A gene that cause Fabry disease. $A m \mathcal{F}$ Hum Genet 1993;53:1186-97.

14 Colombi A, Kostyal A, Bracher R, Gloor F, Mazzi R, Tholen $\mathrm{H}$. Angiokeratoma corporis diffusum - Fabry's disease. Helv Med Acta 1967;34:67-83.

15 Lockman LA, Hunninghake DB, Krivit W, Desnick RJ. Relief of pain of Fabry's disease by diphenylhydantoin. Neurology 1973;23:871-5.

16 Filling-Katz MR, Merrick HF, Fink JK, Miles RB, Sokol J, Barton NW. Carbamazepine in Fabry's disease: effective analgesia with dose-dependent exacerbation of autonomic dysfunction. Neurology 1989;39:598-600.

17 Gordon KE, Ludman MD, Finley GA. Successful treatment of painful crises of Fabry disease with low dose morphine. Pediatr Neurol 1995;12:250-1.

$18 \mathrm{Hilz}$ MJ, Stemper B, Kolodny EH. Lower limb cold exposure induces pain and prolonged small fiber dysfunction in Fabry patients. Pain 2000;84:361-5.

19 Ro LS, Chen ST, Tang LM, Hsu WC, Chang HS, Huang CC. Current perception threshold testing in Fabry's CC. Current perception threshold
disease. Muscle Nerve 1999;22:1531-7.

20 Scott LJ, Griffin JW, Luciano C, Barton NW, Banerjee T, Crawford T, McArthur JC, Tournay A, Schiffmann R. Crawford T, McArthur JC, Tournay A, Schiffmann R.
Quantitative analysis of epidermal innervation in Fabry Quantitative analysis of epidermal
disease. Neurology 1999;52:1249-54.

21 Mitsias P, Levine SR. Cerebrovascular complications of Fabry's disease. Ann Neurol 1996;40:8-17.

22 Grewal RP. Stroke in Fabry's disease. F Neurol 1994;241: 153-6.

23 Grewal RP, Barton NW. Fabry's disease presenting with stroke. Clin Neurol Neurosurg 1992;94:177-9.

24 Tedeschi G, Bonavita S, Banerjee TK, Virta A, Schiffmann R. Diffuse central neuronal involvement in Fabry disease: a
proton MRS imaging study. Neurology 1999;52:1663-7.

25 Crutchfield KE, Patronas NJ, Dambrosia JM, Frei KP, Banerjee TK, Barton NW, Schiffmann R. Quantitative analysis of cerebral vasculopathy in patients with Fabry disease. of cerebral vasculopathy in

26 Grewal RP. Psychiatric disorders in patients with Fabry's disease. Int f Psychiatr Med 1993;23:307-12

27 Tsakiris D, Simpson HK, Jones EH, Briggs JD, Elinder CG, Mendel S, Piccoli G, Dos SJ, Tognoni G, Vanrenterghem $Y$, Valderrabano F. Report on management of renal failure in Europe, XXVI, 1995. Rare diseases in renal replacement therapy in the ERA-EDTA Registry. Nephrol Dial Transplant 1996;11(suppl 7):4-20.

28 Gubler MC, Lenoir G, Grunfeld JP, Ulmann A, Droz D, Habib R. Early renal changes in hemizygous and heterozygous patients with Fabry's disease. Kidney Int 1978;13:223-35.

29 Chatterjee S, Gupta P, Pyeritz RE, Kwiterovich PO Jr. Immunohistochemical localization of glycosphingolipid in urinary renal tubular cells in Fabry's disease. Am $\mathcal{f}$ Clin Pathol 1984;82:24-8.

30 Sirvent AE, Enriquez R, Antolin A, Cabezuelo JB, Teruel A, Rodriguez, Ledesma JM, Gonzalez C, Reyes A. Fabry's 
disease presenting with oligoanuric end-stage renal failure. Nephrol Dial Transplant 1997:12:1503-5.

31 Mehta J, Tuna N, Moller JH, Desnick RJ. Electrocardiographic and vectorcardiographic abnormalities in Fabry's disease. Am Heart f 1977;93:699-705.

32 Goldman ME, Cantor R, Schwartz MF, Baker M, Desnick RJ. Echocardiographic abnormalities and disease severity in Fabry's disease. F Am Coll Cardiol 1986;7:1157-61.

33 Iwase M, Yamauchi K, Maeda M, Aoki T, Yokota M, Hayashi $\mathrm{H}$, Sotobata I. Echocardiographic findings in a case of Fabry's disease with aortic regurgitation and complete AV block, and in his family members (in Japanese). $\mathcal{F}$ Cardiol 1988;18:589-98.

34 Becker AE, Schoorl R, Balk AG, van der Heide RM. Cardiac manifestations of Fabry's disease. Report of a case with mitral insufficiency and electrocardiographic evidence of myocardial infarction. Am 7 Cardiol 1975;36:829-35.

35 Ogawa T, Kawai M, Matsui T, Seo A, Aizawa O, Hongo K, Shiva T, Kawai M, Matsui T, Seo A, Aizawa O, Hongo K, Shibata T, Yoshida S, Okamura T, Nishikawa T, Kasajima showed normal coronary angiographic findings. Fpn Circ $f$ showed normal

36 Elleder M, Dorazilova V, Bradova V, Belohlavek M, Kral V, Choura $M$, Budesinsky $M$, Harzer K. Cardiocyte storage and hypertrophy as a sole manifestation of Fabry disease. Report on a case simulating hypertrophic non-obstructive cardiomyopathy. Virchows Arch A Pathol Anat Histopathol 1990;417:449-55

37 Nagao Y, Nakashima H, Fukuhara Y, Shimmoto M, Oshima A, Ikari Y, Mori Y, Sakuraba H, Suzuki Y. Hypertrophic cardiomyopathy in late-onset variant of Fabry disease with high residual activity of alpha-galactosidase A. Clin Genet 1991;39:233-7.

38 Sheth KJ, Werlin SL, Freeman ME, Hodach AE. Gastrointestinal structure and function in Fabry's disease. $A m \mathcal{F}$ Gastroenterol 1981;76:246-51.
39 Morgan SH, Rudge P, Smith SJ, Bronstein AM, Kendall BE, Holly E, Young EP, Crawfurd MD, Bannister R. The neurological complications of Anderson-Fabry disease (alpha-galactosidase A deficiency) - investigation of symptomatic and presymptomatic patients. $Q \mathcal{F} \mathrm{Med} 1990 ; 75$ : 491-507.

40 Tsukada N, Hanu N, Oguchi K, Yanagisawa N, Tsukagoshi $\mathrm{H}$, Hattori $\mathrm{H}$. Fabry's disease with malocclusion and acromegalic-appearance: clinical and electromicroscopic studies (in Japanese). No to Shinkei 1980;32:311-19.

41 Miners AH, Holmes A, MacDermot KD. Assessment of health-related quality-of-life in males with Anderson Fabry disease before therapeutic intervention. Oual Life (submitted).

42 Miners AH, Sabin CA, Tolley KH, Jenkinson C, Kind P, Lee $\mathrm{CA}$. Assessing health-related quality-of-life in individuals with haemophilia. Haemophilia 1999;5:378-85.

43 Schiffmann R, Kopp JB, Austin HA III, Sabnis S, Moore DF, Weibel T, Balow JE, Brady RO. Enzyme replacement therapy in Fabry disease: a randomized controlled trial. 7AMA 2001;285:2743-9.

44 Cox T, Lachmann R, Hollak C, Aerts J, van Weely S, Hrebicek M, Platt, F, Butters T, Dwek R, Moyses C, Gow I, Elstein D, Zimran A. Novel oral treatment of Gaucher's disease with N-butyldeoxynojirimycin (OGT 918) to decrease substrate biosynthesis. Lancet 2000;355:1481-5.

45 Abe A, Arend LJ, Lee L, Lingwood C, Brady RO, Shayman JA, Schiffmann R, Murray GJ, Treco D, Daniel P, Sellos-Moura M, Myers M, Quirk JM, Zirzow GC, Borowski M, Loveday K, Anderson T, Gillespie F, Oliver KL, Jeffries NO, Doo E, Liang TJ, Kreps C, Gunter K, Frei K, Crutchfield K, Selden RF, Brady RO. Glycosphingolipid depletion in Fabry disease lymphoblasts with potent inhibitors of glucosylceramide synthase. Kidney Int 2000; 57:446-54.

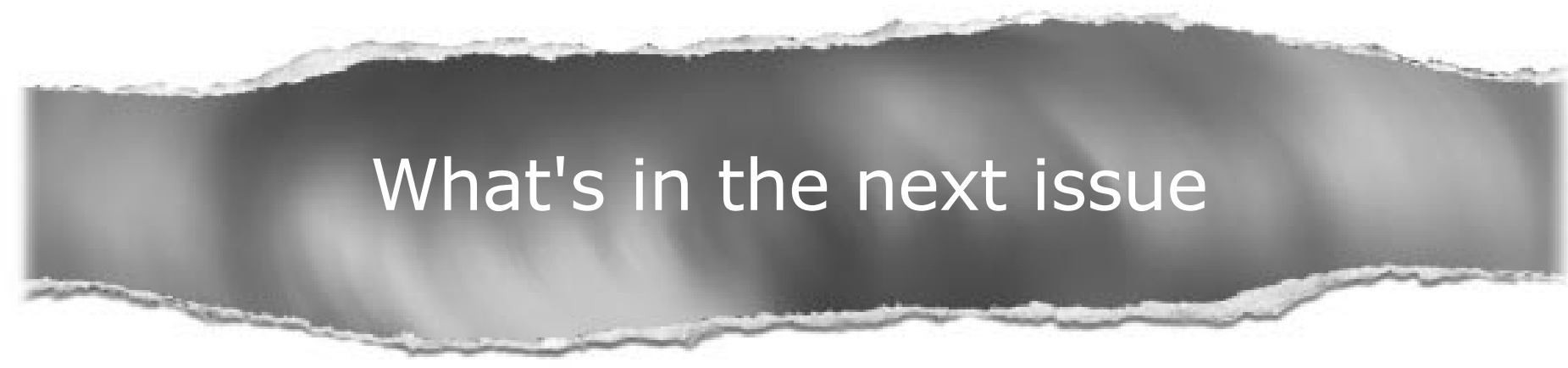

Future content

See which articles have just been accepted for publication and preview the table of contents for the next issue a month before it is published

\section{wWw.jmedgenet.com}

